Article

\title{
Application of UHPLC-ESI-Q-TOF-MS to Identify Multiple Constituents in Processed Products of the Herbal Medicine Ligustri Lucidi Fructus
}

\author{
Hui Li, Weifeng Yao *, Qinan Liu, Jia Xu, Beihua Bao, Mingqiu Shan, Yudan Cao, \\ Fangfang Cheng, Anwei Ding and Li Zhang * \\ Jiangsu Collaborative Innovation Center of Chinese Medicinal Resources Industrialization, \\ Jiangsu Key Laboratory for High Technology Research of TCM Formulae, National and Local Collaborative \\ Engineering Center of Chinese Medicinal Resources Industrialization and Formulae Innovative Medicine, \\ and State Key Laboratory Cultivation Base for TCM Quality and Efficacy, School of Pharmacy, \\ Nanjing University of Chinese Medicine, Nanjing 210023, Jiangsu, China; missyhui2012@163.com (H.L.); \\ liuqinan0728@163.com (Q.L.); njxj0106@126.com (J.X.); scotter01@163.com (B.B.); shanmingqiu@163.com (M.S.); \\ raindc@163.com (Y.C.); cff19870524@163.com (F.C.); awding105@163.com (A.D.) \\ * Correspondence: yaowf@njucm.edu.cn (W.Y.); zhangli@njucm.edu.cn (L.Z.); \\ Tel.: +86-25-8581-1053 (W.Y.); +86-25-8581-1509 (L.Z.); Fax: +86-25-8581-1524 (W.Y. \& L.Z.)
}

Academic Editor: Derek J. McPhee

Received: 9 March 2017; Accepted: 21 April 2017; Published: 26 April 2017

\begin{abstract}
Ligustri Lucidi Fructus (LLF), the fruit of Ligustrum lucidum Ait. (Oleaceae), has been used as a common herbal medicine in clinical practice in China for nearly 2000 years. In most cases, LLF is prescribed in decoctions in the form of processed products rather than crude drugs. In this study, an ultra-high performance liquid chromatography coupled with electrospray ionization-quadrupole-time of flight-mass spectrometry (UHPLC-ESI-Q-TOF-MS) method was established for rapid separation and identification of multiple constituents in the $80 \%$ methanol extract of processed-LLF. A total of 50 compounds (one phenylethanoid, seven phenylethanoid glycosides, seven flavonoids, 25 iridoids, nine triterpenoids and one cyclohexanecarboxylic acid) were either unambiguously identified or tentatively characterized with the aid of authentic standards or published data. Luteolin-7-O-rutinoside, oleoside and secologanoside were detected in LLF for the first time. This study enriches the chemical profiling of processed-LLF and could provide valuable information for the quality control and further investigation of processed-LLF and crude LLF.
\end{abstract}

Keywords: processed-Ligustri Lucidi Fructus; UHPLC-ESI-Q-TOF-MS; phenylethanoids; flavonoids; secoiridoids; triterpenoids

\section{Introduction}

Ligustri Lucidi Fructus (LLF), the dried ripe fruit of Ligustrum lucidum Ait. (Oleaceae), also known as Nüzhenzi in Traditional Chinese Medicine (TCM), is not only a widely used herbal medicine in China, but also a functional food material authorized by the China Food and Drug Administration (CFDA). Nüzhenzi was first recorded as a traditional Chinese medicine in the earliest book of Chinese herbal medicine, titled Shen Nong's Herbal Classic [1], which was written in the Western Han Dynasty and has a history of almost 2000 years of use. In this ancient and classical book, Nüzhenzi was listed in the "top class" medicines and claimed to have the ability of maintaining healthy energy. According to the 2015 edition of Chinese Pharmacopoeia, the main function of Nüzhenzi is to nourish liver and kidney, brighten eyes, and make the hair black [2]. Modern research on LLF has demonstrated that various extracts and individual compounds derived from this herbal medicine exhibit extensive bioactivities, such as hepatoprotective, anti-tumor, anti-osteoporosis, antioxidant, anti-inflammatory, 
anti-ageing, immune regulation, etc. [3]. In clinical practice, crude drugs usually need to be treated in some special way before they can be used as medicines, which is one of the characteristic features of TCMs. In the case of LLF, there are many processing methods, e.g., steaming with wine, vinegar, salt solution or the juice of other herbs, among which, wine steaming is the most important one and has been recorded in the Chinese Pharmacopoeia. After being steamed with wine, the function of LLF will be strengthened and the chemical constituents will change, not only in quantity but also in quality [4]. Over the past few years, several types of chemical constituents have been isolated from LLF, including phenylethanoid glycosides (PhGs), flavonoids, iridoids, triterpenoids and other components such as polysaccharides, amino acids, fatty acids and some minor elements [3]. Ligustrum lucidum Ait. is widely distributed in China, and is often used for environmental greening in many places, which makes the source of LLF very abundant. In the view of the medicinal, edible and chemical values, LLF has a great development potential.

Recently, UHPLC-ESI-Q-TOF-MS has become a powerful tool in the characterization of complex natural products and been widely used in TCM research [5-7]. ESI is a soft ionization method capable of providing both protonated and deprotonated molecules. Q-TOF-MS is able to combine high sensitivity and mass accuracy for both precursor and product ions, and therefore makes it possible to confirm the elemental composition for both parent and fragment ions in a rapid and efficient way [8]. Meanwhile, the application of UHPLC can provide high resolution for the separation of complicated natural products and improve the sensitivity of a Q-TOF-MS detector [9].

To the best of our knowledge, some constituents, e.g., iridiod glycosides, have been identified in LLF and analyzed using ESI-Q-TOF-MS [10]. However, the comprehensive identification of multiple constituents in processed-LLF has not been reported. Therefore, we decided to investigate the multiple constituents in processed-LLF through UHPLC-ESI-Q-TOF-MS, thus providing in-depth knowledge of its chemical composition and offering valuable information for its quality control and further study.

\section{Results and Discussion}

\subsection{UHPLC-ESI-Q-TOF-MS Analysis of Constituents in Processed-LLF}

In this study, most of the compounds showed higher response in the negative mode than in the positive mode. Therefore, negative ion mode has been employed to identify the corresponding signals. The identification of compounds was carried out by comparing their retention times and mass spectra provided by TOF-MS with those of authentic standards when available. The remaining compounds, for which no commercial standards available, were characterized by the interpretation of their mass spectra and the information previously reported in the literature. Figure 1 illustrates the base peak chromatogram (BPC) of the processed-LLF extract in ESI negative mode. As shown in Table 1 and Figure 2, a total of 50 compounds were either unambiguously identified (six compounds) or tentatively characterized (44 compounds).

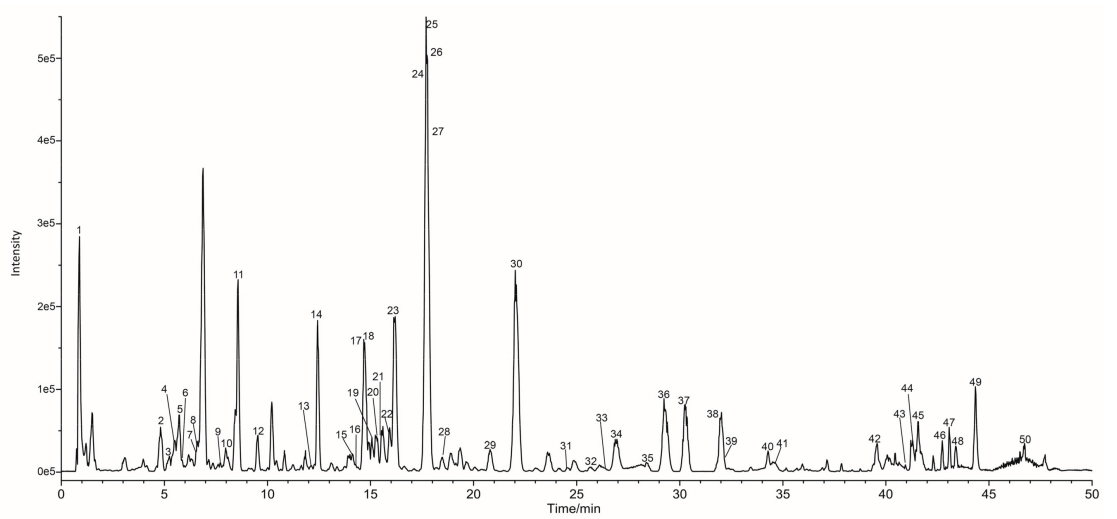

Figure 1. Base Peak Chromatogram (BPC) in negative mode of processed-LLF. 
Table 1. Identification of 51 constituents of processed-LLF by UHPLC-ESI-Q-TOF-MS/MS in negative mode.

\begin{tabular}{|c|c|c|c|c|c|c|c|c|}
\hline \multirow{2}{*}{ Peak } & \multirow{2}{*}{$\mathrm{RT}(\min )$} & \multirow{2}{*}{ Formula } & \multicolumn{3}{|c|}{$\left[\mathbf{M}-\mathbf{H}^{-}\right.$} & \multirow{2}{*}{ MS $^{2}$ Fragments (Relative Abundance) } & \multirow{2}{*}{ Proposed Compound } & \multirow{2}{*}{ Ref. } \\
\hline & & & $m / z$ theo & $m / z \exp$ & Error (ppm) & & & \\
\hline \multicolumn{9}{|c|}{ Phenylethanoids } \\
\hline 2 & 4.81 & $\mathrm{C}_{8} \mathrm{H}_{10} \mathrm{O}_{3}$ & 153.0557 & 153.0556 & -1 & $123.0456(100)$ & Hydroxytyrosol & [11] \\
\hline 3 & 5.22 & $\mathrm{C}_{14} \mathrm{H}_{20} \mathrm{O}_{8}$ & 315.1085 & 315.1089 & 1.1 & 153.0554 (94.24), $123.0454(100)$ & Hydroxytyrosol Glucoside & [11] \\
\hline 7 & 6.60 & $\mathrm{C}_{14} \mathrm{H}_{20} \mathrm{O}_{7}$ & 299.1136 & 299.1140 & 1.2 & 137.0587 (13.60), $119.0500(83.73), 113.0250(27.20), 101.0270(24.53), 59.0194$ (100) & Salidroside & standard \\
\hline 9 & 7.72 & $\mathrm{C}_{19} \mathrm{H}_{28} \mathrm{O}_{11}$ & 431.1559 & 431.1558 & -0.1 & 299.1114 (10.82), 149.0457 (25.15), 119.0486 (12.54), 101.0255 (12.87) & Osmanthuside $\mathrm{H}$ & [12] \\
\hline 13 & 12.13 & $\mathrm{C}_{29} \mathrm{H}_{36} \mathrm{O}_{16}$ & 639.1931 & 639.1933 & 0.4 & 621.1878 (47.63), $459.1506(10.31), 179.0341(69.58), 161.0238$ (100), 135.0447 (12.63) & $\beta$-Hydroxyverbascoside & [13] \\
\hline 14 & 12.44 & $\mathrm{C}_{35} \mathrm{H}_{46} \mathrm{O}_{20}$ & 785.2510 & 785.2517 & 0.9 & 623.2239 (39.09), $461.1683(2.63), 179.0371$ (2.79), 161.0236 (35.63), 135.0450 (3.32) & Echinacoside & [5] \\
\hline 23 & 16.18 & $\mathrm{C}_{29} \mathrm{H}_{36} \mathrm{O}_{15}$ & 623.1981 & 623.1989 & 1.3 & 461.1669 (35.12), 161.0234 (100), 179.0342 (7.52), 135.0447 (13.66) & Verbascoside & standard \\
\hline 26 & 17.81 & $\mathrm{C}_{29} \mathrm{H}_{36} \mathrm{O}_{15}$ & 623.1981 & 623.1983 & 0.3 & $461.1679(34.39), 161.0235(100), 179.0340(6.70), 135.0448(12.00)$ & Isoverbascoside & [5] \\
\hline \multicolumn{9}{|c|}{$\begin{array}{ll} & \text { Flavonoids } \\
\end{array}$} \\
\hline 16 & 14.39 & $\mathrm{C}_{27} \mathrm{H}_{30} \mathrm{O}_{16}$ & 609.1461 & 609.1466 & 0.8 & 301.0356 (39.90), 300.0278 (89.95), 178.9991 (4.59), 151.0033 (4.34) & Quercetin-3-O-rutinoside & {$[8,14]$} \\
\hline 18 & 14.78 & $\mathrm{C}_{27} \mathrm{H}_{30} \mathrm{O}_{15}$ & 593.1512 & 593.1511 & -0.2 & $285.0407(100), 284.0327(9.31)$ & Luteolin-7-O-rutinoside & [15] \\
\hline 20 & 15.28 & $\mathrm{C}_{21} \mathrm{H}_{20} \mathrm{O}_{11}$ & 447.0933 & 447.0934 & 0.2 & 285.0397 (100), $284.0320(44.59)$ & Luteolin-7-O-glucoside & standard \\
\hline 24 & 17.60 & $\mathrm{C}_{27} \mathrm{H}_{30} \mathrm{O}_{14}$ & 577.1563 & 577.1557 & -1 & $311.0559(0.57), 269.0450(100)$ & Apigenin-7-O-rutinoside & [16] \\
\hline 28 & 18.48 & $\mathrm{C}_{21} \mathrm{H}_{20} \mathrm{O}_{10}$ & 431.0984 & 431.0983 & -0.1 & $269.0454(26.47), 268.0369(100)$ & Apigenin-7-O-glucoside & [17] \\
\hline 33 & 26.33 & $\mathrm{C}_{15} \mathrm{H}_{10} \mathrm{O}_{6}$ & 285.0405 & 285.0408 & 1.2 & $151.0033(14.30), 133.0297(68.21)$ & Luteolin & standard \\
\hline 39 & 32.14 & $\mathrm{C}_{15} \mathrm{H}_{10} \mathrm{O}_{5}$ & 269.0456 & 269.0455 & -0.2 & 151.0041 (14.36), 117.0353 (58.84) & Apigenin & standard \\
\hline \multicolumn{9}{|c|}{ Iridoids } \\
\hline 4 & 5.50 & $\mathrm{C}_{16} \mathrm{H}_{22} \mathrm{O}_{11}$ & 389.1089 & 389.1093 & 0.9 & 345.1190 (3.03), 227.0557 (37.80), 209.0454 (14.54), 183.0660 (87.28), 165.0551 (67.05), 121.0665 (100) & Oleoside & {$[13,18]$} \\
\hline 5 & 5.71 & $\mathrm{C}_{16} \mathrm{H}_{24} \mathrm{O}_{10}$ & 375.1297 & 375.1300 & 0.9 & $331.1405(70.23), 169.0872(3.45), 151.0761(25.68), 213.0783(1.16), 113.0250(39.00)$ & Loganic acid & {$[11,19]$} \\
\hline 6 & 5.84 & $\mathrm{C}_{17} \mathrm{H}_{24} \mathrm{O}_{14}$ & 451.1093 & 451.1092 & -0.3 & $\begin{array}{l}\begin{array}{l}433.0995(11.52), 271.0457(22.27), 239.0177(14.00), 227.0544(28.26), 195.0296(70.07), 183.0653 \\
(25.05), 151.0398(100), 123.0459(35.06)\end{array}\end{array}$ & Nuezhenidic acid & [20] \\
\hline 8 & 6.61 & $\mathrm{C}_{10} \mathrm{H}_{14} \mathrm{O}_{5}$ & 213.0768 & 213.0777 & 4.1 & 183.0666 (100), $151.0768(54.45), 121.0670(31.32), 107.0889(9.07)$ & Nuzhenal A & [21] \\
\hline 10 & 8.06 & $\mathrm{C}_{17} \mathrm{H}_{24} \mathrm{O}_{11}$ & 403.1246 & 403.1247 & 0.3 & $\begin{array}{l}223.0598 \text { (54.55), 179.0555 (26.50), } 121.0307 \text { (41.08), } 119.0355 \text { (57.87), } 113.0242 \text { (34.44), } 101.0249 \\
(55.77), 89.0264(97.55), 59.0190(100)\end{array}$ & Oleoside 11-methyl ester (isomer) & {$[8,11,22]$} \\
\hline 11 & 8.57 & $\mathrm{C}_{16} \mathrm{H}_{22} \mathrm{O}_{11}$ & 389.1089 & 389.1093 & 0.9 & 345.1192 (29.00), 209.0449 (17.65), 183.0658 (26.15), 165.0552 (41.48), 121.0656 (62.61) & Secologanoside & {$[13,18,19]$} \\
\hline 12 & 9.53 & $\mathrm{C}_{17} \mathrm{H}_{24} \mathrm{O}_{11}$ & 403.1246 & 403.1250 & 1 & $\begin{array}{l}223.0608(47.43), 179.0564(26.79), 121.0290(25.08), 119.0360 \text { (52.49), } 113.0251 \text { (30.06), } 101.0266 \\
(56.07), 89.0270(100), 59.0193(83.41)\end{array}$ & Oleoside 11-methyl ester & {$[8,11,22]$} \\
\hline 15 & 14.12 & $\mathrm{C}_{25} \mathrm{H}_{32} \mathrm{O}_{14}$ & 555.1719 & 555.1725 & 1 & 323.0771(3.64), 223.0603(7.70), 151.0397(100), 123.0453(11.68) & 10-Hydroxyoleuropein & {$[8,23]$} \\
\hline 17 & 14.71 & $\mathrm{C}_{25} \mathrm{H}_{30} \mathrm{O}_{15}$ & 569.1512 & 569.1517 & 0.9 & $389.0899(30.33), 209.0452(46.58), 151.0402(100), 123.0455(26.25)$ & Oleuropeinic acid & {$[24,25]$} \\
\hline 19 & 15.07 & $\mathrm{C}_{31} \mathrm{H}_{42} \mathrm{O}_{18}$ & 701.2298 & 701.2311 & 1.8 & $539.1797(12.19), 469.1375(20.41), 437.1443(10.17), 315.1081(100)$ & Neonuzhenide & [26] \\
\hline 21 & 15.48 & $\mathrm{C}_{11} \mathrm{H}_{14} \mathrm{O}_{6}$ & 241.0718 & 241.0724 & 2.5 & 139.0035 (100), $127.0403(54.59), 121.0295(27.57), 101.0262(44.93), 95.0522$ (61.86) & Elenolic acid & {$[27,28]$} \\
\hline 22 & 15.94 & $\mathrm{C}_{31} \mathrm{H}_{42} \mathrm{O}_{17}$ & 685.2349 & 685.2360 & 1.6 & $\begin{array}{l}\text { 523.1853 (75.34), } 453.1422 \text { (98.87), } 421.1519 \text { (53.10), } 299.1134 \text { (100), } 223.0606 \text { (82.17), } 179.0558 \\
(29.48), 119.0371 \text { (31.24) }\end{array}$ & Nuezhenide (isomer) & - \\
\hline
\end{tabular}


Table 1. Cont

\begin{tabular}{|c|c|c|c|c|c|c|c|c|}
\hline \multirow[b]{2}{*}{ Peak } & \multirow{2}{*}{$\mathrm{RT}(\min )$} & \multirow{2}{*}{ Formula } & \multicolumn{3}{|c|}{$[\mathbf{M}-\mathbf{H}]^{-}$} & \multirow{2}{*}{ MS $^{2}$ Fragments (Relative Abundance) } & \multirow{2}{*}{ Proposed Compound } & \multirow{2}{*}{ Ref. } \\
\hline & & & $m / z$ theo & $m / z \exp$ & Error (ppm) & & & \\
\hline \multicolumn{9}{|c|}{ Iridoids } \\
\hline 25 & 17.72 & $\mathrm{C}_{31} \mathrm{H}_{42} \mathrm{O}_{17}$ & 685.2349 & 685.2356 & 0.9 & $\begin{array}{l}\text { 523.1818 (30.54), } 453.1395 \text { (100),421.1483 (49.59), } 299.1117 \text { (59.94), } 223.0593 \text { (51.80), } 179.0557 \\
(21.62), 119.0374(17.98)\end{array}$ & Specnuezhenide & standard \\
\hline 27 & 17.83 & $\mathrm{C}_{25} \mathrm{H}_{30} \mathrm{O}_{14}$ & 553.1563 & 553.1558 & -0.8 & $\begin{array}{l}\begin{array}{l}509.1670 \\
(82.67)\end{array} \\
(11.95), 477.1435(4.16), 391.1015(8.35), 373.0941 \text { (41.57), } 347.1143(100), 209.0447\end{array}$ & Ligustrosidic acid & {$[10,25]$} \\
\hline 29 & 20.80 & $\mathrm{C}_{31} \mathrm{H}_{42} \mathrm{O}_{17}$ & 685.2349 & 685.2356 & 0.9 & $\begin{array}{l}523.1856(24.96), 453.1429 \text { (44.64), } 421.1518(22.12), 385.1150 \text { (22.09), } 299.1134 \text { (100), } 223.0609 \\
(24.93), 179.0555 \text { (20.58), } 119.0364(25.63)\end{array}$ & Isonuezhenide & [26] \\
\hline 30 & 22.06 & $\mathrm{C}_{25} \mathrm{H}_{32} \mathrm{O}_{13}$ & 539.1770 & 539.1773 & 0.5 & $\begin{array}{l}\text { 403.1253 (14.23), 377.1241 (57.51), } 307.0813 \text { (91.75), } 275.0877 \text { (79.24), } 223.0601 \text { (46.21), } 179.0563 \\
(19.28), 149.0241 \text { (100), } 139.0381 \text { (54.09) }\end{array}$ & Oleuropein & {$[8,11,29]$} \\
\hline 31 & 24.56 & $\mathrm{C}_{48} \mathrm{H}_{64} \mathrm{O}_{27}$ & 1071.3562 & 1071.3581 & 1.7 & $\begin{array}{l}909.3186(10.15), 839.2691 \text { (9.04), } 771.2401(31.56), 685.2395(36.40), 523.1839(26.89), 453.1413 \\
(29.53), 403.1255(18.24), 385.1166(12.15), 299.1139(11.67), 223.0605(23.17)\end{array}$ & G 13 & {$[13,30]$} \\
\hline 32 & 25.68 & $\mathrm{C}_{27} \mathrm{H}_{36} \mathrm{O}_{14}$ & 583.2032 & 583.2035 & 0.5 & $537.1649(31.84), 403.1255$ (35.57), $223.0603(32.74), 151.0401$ (100) & Lucidumoside $\mathrm{C}$ & [8] \\
\hline 34 & 26.91 & $\mathrm{C}_{25} \mathrm{H}_{32} \mathrm{O}_{12}$ & 523.1821 & 523.1820 & -0.2 & $361.1296(27.22), 291.0870(100), 259.0968(22.10), 101.0260$ (20.27) & Ligustroside & {$[8,10,31]$} \\
\hline 35 & 28.41 & $\mathrm{C}_{48} \mathrm{H}_{64} \mathrm{O}_{27}$ & 1071.3562 & 1071.3585 & 2.1 & $\begin{array}{l}909.3161(22.61), 839.2736(16.57), 771.2440(20.00), 685.2429(45.75), 523.1855(35.60), 453.1438 \\
(23.63), 403.1267(14.76), 385.1145 \text { (6.14), } 299.1135(13.15), 223.0603(117.03)\end{array}$ & G 13 (isomer) & {$[13,30]$} \\
\hline 36 & 29.28 & $\mathrm{C}_{25} \mathrm{H}_{28} \mathrm{O}_{12}$ & 519.1508 & 519.1512 & 0.7 & $\begin{array}{l}227.0560(13.80), 189.0557 \text { (34.00), } 183.0664(34.60), 165.0557(28.12), 161.0610 \text { (100), } 147.0457 \\
(63.08), 121.0669(46.70)\end{array}$ & 6'-O-trans-Cinnamoyl-8-epikingisidic acid & [21] \\
\hline 37 & 30.27 & $\mathrm{C}_{48} \mathrm{H}_{64} \mathrm{O}_{27}$ & 1071.3562 & 1071.3578 & 1.4 & $\begin{array}{l}909.3159 \text { (15.90), } 839.2724(12.16), 771.2436(52.95), 685.2404(100), 523.1850(63.76), 453.1421 \\
(44.96), 403.1256(28.24), 385.1157(15.01), 299.1137(28.75), 223.0611(37.36)\end{array}$ & G 13 (isomer) & {$[13,30]$} \\
\hline 38 & 31.99 & $\mathrm{C}_{48} \mathrm{H}_{64} \mathrm{O}_{27}$ & 1071.3562 & 1071.3583 & 1.9 & $\begin{array}{l}909.3147(38.95), 839.2689(30.69), 771.2422(23.33), 685.2387(90.89), 523.1830(61.19), 453.1407 \\
(42.67), 403.1256(25.28), 385.1148(7.88), 399.1131(22.25), 223.0604(30.57)\end{array}$ & G 13 (isomer) & {$[13,30]$} \\
\hline 40 & 34.28 & $\mathrm{C}_{25} \mathrm{H}_{28} \mathrm{O}_{12}$ & 519.1508 & 519.1510 & 0.3 & $\begin{array}{l}\text { 475.1626 (29.18), } 209.0447 \text { (14.46), } 189.0552 \text { (41.69), } 183.0654 \text { (19.17), } 165.0565 \text { (29.89), } 161.0604 \\
(100), 147.0448(76.02), 121.0667(42.59)\end{array}$ & 6'-O-cis-Cinnamoyl 8-epikingisidic acid & [21] \\
\hline 41 & 34.54 & $\mathrm{C}_{19} \mathrm{H}_{22} \mathrm{O}_{8}$ & 377.1242 & 377.1243 & 0.2 & 307.0800 (39.23), $275.0899(25.15), 149.0273(100), 139.0394(77.64)$ & Oleuropein aglycone & {$[27,29,32]$} \\
\hline \multicolumn{9}{|c|}{ Triterpenes } \\
\hline 42 & 39.55 & $\mathrm{C}_{30} \mathrm{H}_{48} \mathrm{O}_{5}$ & 487.3429 & 487.3424 & -1 & $469.3340(18.52), 423.3282(15.84)$ & Tormentic acid & [33] \\
\hline 43 & 40.97 & $\mathrm{C}_{30} \mathrm{H}_{48} \mathrm{O}_{4}$ & 471.3480 & 471.3473 & -1.9 & 453.3402 (60.01), 407.3328 (18.05), 451.3225 (13.04) & $19 \alpha$-Hydroxyursolic acid & [33] \\
\hline 44 & 41.30 & $\mathrm{C}_{30} \mathrm{H}_{48} \mathrm{O}_{4}$ & 471.3480 & 471.3476 & -0.8 & - & $2 \alpha$-Hydroxyoleanolic acid & [33] \\
\hline 45 & 41.56 & $\mathrm{C}_{30} \mathrm{H}_{48} \mathrm{O}_{4}$ & 471.3480 & 471.3475 & -0.9 & - & $2 \alpha$-Hydroxyursolic acid & [3] \\
\hline 46 & 42.73 & $\mathrm{C}_{39} \mathrm{H}_{54} \mathrm{O}_{6}$ & 617.3848 & 617.3846 & -0.2 & $145.0292(14.69)$ & $3 \beta$-O-trans- $p$-Coumaroylmaslinic acid & [34] \\
\hline 47 & 43.08 & $\mathrm{C}_{39} \mathrm{H}_{54} \mathrm{O}_{6}$ & 617.3848 & 617.3847 & -0.1 & $145.0288(17.35)$ & $3 \beta$-O-cis- $p$-Coumaroylmaslinic acid & [34] \\
\hline 48 & 43.39 & $\mathrm{C}_{32} \mathrm{H}_{50} \mathrm{O}_{5}$ & 513.3586 & 513.3579 & -1.2 & $495.3497(27.14), 453.3390(3.71)$ & $19 \alpha$-Hydroxy-3-acetylursolic acid & [34] \\
\hline 49 & 44.35 & $\mathrm{C}_{30} \mathrm{H}_{48} \mathrm{O}_{3}$ & 455.3531 & 455.3526 & -1 & - & Oleanolic acid/Ursolic acid & standard \\
\hline 50 & 46.71 & $\mathrm{C}_{32} \mathrm{H}_{50} \mathrm{O}_{4}$ & 497.3636 & 497.3632 & -0.9 & - & Acetyloleanolic acid/Acetylursolic acid & [34] \\
\hline \multicolumn{9}{|c|}{ Other Compounds } \\
\hline 1 & 0.88 & $\mathrm{C}_{7} \mathrm{H}_{12} \mathrm{O}_{6}$ & 191.0561 & 191.0569 & 4.2 & 173.0447 (11.54), 127.0394 (20.92), 109.0301 (13.44), 93.0359 (67.79), 85.0314 (100) & Quinic acid & {$[8,19]$} \\
\hline
\end{tabular}


Phenylethanoids<smiles>[R]Oc1ccc(CCO)cc1O</smiles>

(2) $\mathrm{R}_{1}=\mathrm{H}$

(3) $R_{1}=G l c$<smiles>CC1OC[C@](O)(CO)[C@H]1O</smiles><smiles>[R9]C[C@H]1O[C@H](OCCc2ccc(O)cc2)[C@@H](O)[C@H](O)[C@H]1O</smiles><smiles>CC(=O)C=Cc1ccc(O)c(O)c1</smiles><smiles>[R6]CC1OC(OCC([R6])c2ccc(O)c(O)c2)[C@H](O)[C@@H](C([R6])[R6])[C@H]1O</smiles>

(13) $\mathrm{R}_{1}=\mathrm{OH}, \mathrm{R}_{2}=$ Rha, $\mathrm{R}_{3}=$ Caff, $\mathrm{R}_{4}=\mathrm{H}$

(14) $R_{1}=H, R_{2}=$ Rha, $R_{3}=$ Caff, $R_{4}=G l c$

(23) $\mathrm{R}_{1}=\mathrm{H}, \mathrm{R}_{2}=\mathrm{Rha}, \mathrm{R}_{3}=$ Caff, $\mathrm{R}_{4}=\mathrm{H}$

(26) $\mathrm{R}_{1}=\mathrm{H}, \mathrm{R}_{2}=\mathrm{Rha}, \mathrm{R}_{3}=\mathrm{H}, \mathrm{R}_{4}=$ Caff
Flavonoids<smiles>[R6]Oc1cc(O)c2c(=O)c([R9])c(-c3ccc([R6])c([R])c3)oc2c1</smiles>

(16) $\mathrm{R}_{1}=\mathrm{OH}, \mathrm{R}_{2}=\mathrm{H}, \mathrm{R}_{3}=$ Rutinose, $\mathrm{R}_{4}=\mathrm{H}$ (18) $\mathrm{R}_{1}=\mathrm{OH}, \mathrm{R}_{2}=\mathrm{H}, \mathrm{R}_{3}=\mathrm{H}, \mathrm{R}_{4}=$ Rutinose

(20) $\mathrm{R}_{1}=\mathrm{OH}, \mathrm{R}_{2}=\mathrm{H}, \mathrm{R}_{3}=\mathrm{H}, \mathrm{R}_{4}=\mathrm{Glc}$

(24) $\mathrm{R}_{1}=\mathrm{H}, \mathrm{R}_{2}=\mathrm{H}, \mathrm{R}_{3}=\mathrm{H}, \mathrm{R}_{4}=$ Rutinose

(28) $\mathrm{R}_{1}=\mathrm{H}, \mathrm{R}_{2}=\mathrm{H}, \mathrm{R}_{3}=\mathrm{H}, \mathrm{R}_{4}=\mathrm{GlC}$

(33) $\mathrm{R}_{1}=\mathrm{OH}, \mathrm{R}_{2}=\mathrm{H}, \mathrm{R}_{3}=\mathrm{H}, \mathrm{R}_{4}=\mathrm{H}$

(39) $\mathrm{R}_{1}=\mathrm{H}, \mathrm{R}_{2}=\mathrm{H}, \mathrm{R}_{3}=\mathrm{H}, \mathrm{R}_{4}=\mathrm{H}$

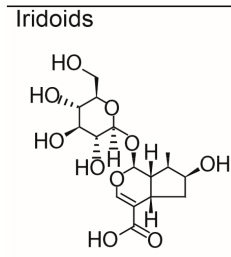

(5) Loganic acid

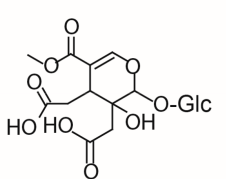

(6) Nuezhenidic acid<smiles>CC=C(C=O)C(CC(=O)O)CC(=O)OC</smiles><smiles>[R]OC(=O)C1=COC(O[C@H]2O[C@H](CO)[C@@H](O)[C@H](O)[C@H]2O)C(C(=O)O)C1C([R])=O</smiles>

(4) $\mathrm{R}_{1}=\mathrm{H}, \mathrm{R}_{2}=\mathrm{H}$

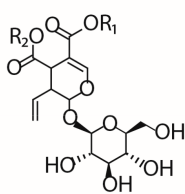

$\begin{array}{llll}\text { (8) Nuzhenal } A & \begin{array}{l}\text { (4) } \mathrm{R}_{1}=\mathrm{H}, \mathrm{R}_{2}=\mathrm{H} \\ \text { (12) } \mathrm{R}_{1}=\mathrm{CH}_{3}, \mathrm{R}_{2}=H\end{array} & \text { (11) } \mathrm{R}_{1}=\mathrm{H}, \mathrm{R}_{2}=\mathrm{H} & \text { (21) Elenolic acid }\end{array}$

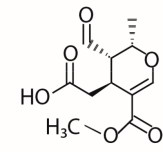

(15) $\mathrm{R}_{1}=\mathrm{CH}_{3}, \mathrm{R}_{2}=\mathrm{Glc}, \mathrm{R} 3=\mathrm{CH}_{2} \mathrm{OH}, \mathrm{R}_{4}=\mathrm{H}, \mathrm{R}_{5}=\mathrm{OH}$

(17) $\mathrm{R}_{1}=\mathrm{CH}_{3}, \mathrm{R}_{2}=\mathrm{Glc}, \mathrm{R}_{3}=\mathrm{COOH}, \mathrm{R}_{4}=\mathrm{H}, \mathrm{R}_{5}=\mathrm{OH}$

(27) $\mathrm{R}_{1}=\mathrm{CH}_{3}, \mathrm{R}_{2}=\mathrm{Glc}, \mathrm{R}_{3}=\mathrm{COOH}, \mathrm{R}_{4}=\mathrm{H}, \mathrm{R}_{5}=\mathrm{H}$

(30) $\mathrm{R}_{1}=\mathrm{CH}_{3}, \mathrm{R}_{2}=\mathrm{Glc}, \mathrm{R}_{3}=\mathrm{CH}_{3}, \mathrm{R}_{4}=\mathrm{H}, \mathrm{R}_{5}=\mathrm{OH}$

$$
\text { (n) }
$$

(19) $\mathrm{R}_{1}=\mathrm{H}, \mathrm{R}_{2}=\mathrm{CH}_{3}, \mathrm{R}_{3}=\mathrm{A}\left(\mathrm{R}^{\prime}=\mathrm{OH}, \mathrm{Glc}^{6}-\mathrm{O}-\right)$

(22) $\mathrm{R}_{1}=\mathrm{CH}_{3}, \mathrm{R}_{2}=\mathrm{H}, \mathrm{R}_{3}=\mathrm{A}\left(\mathrm{R}^{\prime}=\mathrm{H}, \mathrm{Glc}^{4}-\mathrm{O}-\right)$

(25) $\mathrm{R}_{1}=\mathrm{H}, \mathrm{R}_{2}=\mathrm{CH}_{3}, \mathrm{R}_{3}=\mathrm{A}\left(\mathrm{R}^{\prime}=\mathrm{H}, \mathrm{Glc}^{6}-\mathrm{O}-\right)$

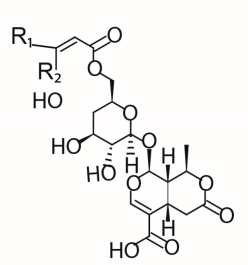

(36) $\mathrm{R}_{1}=\mathrm{Ph}, \mathrm{R}_{2}=\mathrm{H}$ (40) $\mathrm{R}_{1}=\mathrm{H}, \mathrm{R}_{2}=\mathrm{Ph}$

(41) $\mathrm{R}_{1}=\mathrm{CH}_{3}, \mathrm{R}_{2}=\mathrm{H}, \mathrm{R}_{3}=\mathrm{CH}_{3}, \mathrm{R}_{4}=\mathrm{H}, \mathrm{R}_{5}=\mathrm{OH}$

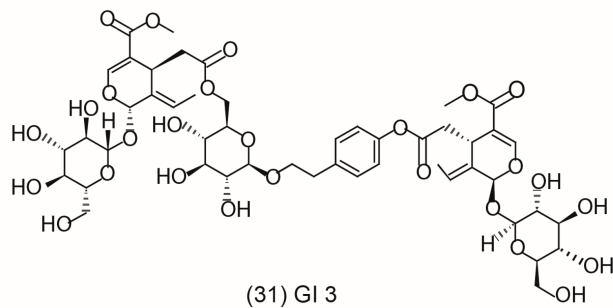

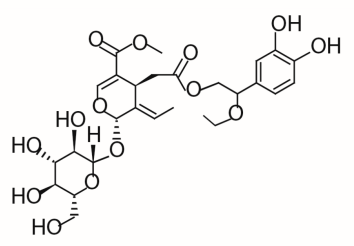

(32) Lucidumoside C

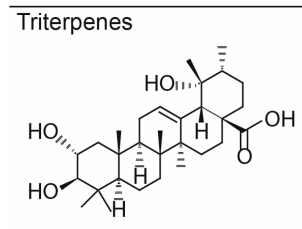

(42) Tormentic acid

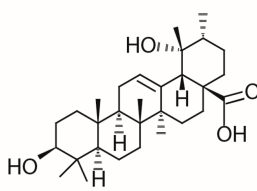

(43) 19a-Hydroxyursolic acid

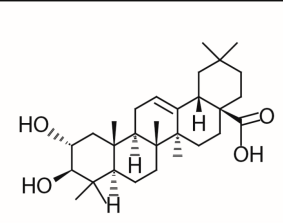

(44) 2a-Hydroxyoleanolic acid

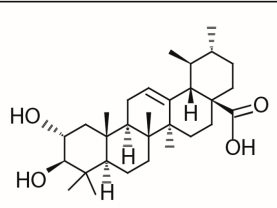

(45) 2a-Hydroxyursolic acid

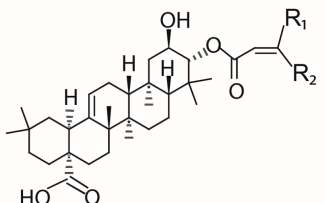

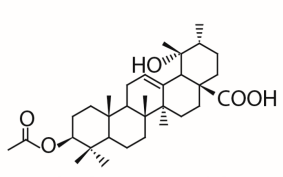

(48) 19a-hydroxy-3-acetyl-ursolic acid

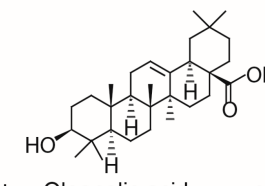

Oleanolic acid or

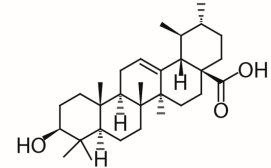

or
$(49)$

(46) $\mathrm{R}_{1}=\mathrm{H}, \mathrm{R}_{2}=\mathrm{p}$-coumaroyl (47) $\mathrm{R}_{1}=\mathrm{p}$-coumaroyl, $\mathrm{R}_{2}=\mathrm{H}$

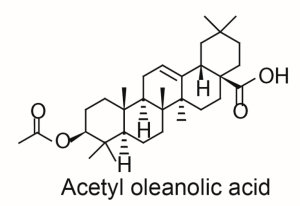

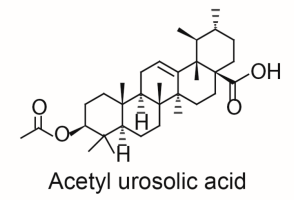

$(50)$

Figure 2. Chemical structures of main compounds identified from processed-LLF. (Glc: $\beta$-D-glucopyranosyl; Rha: Rhamnosyl; Caff: Caffeyl; Api: $\beta$-D-apiofuranosy). 


\subsection{Identification of Phenylethanoids and Glycosides 2, 3, 7, 9, 13, 14, 23, 26}

Phenylethanoid glycosides (PhGs) are an important class of compounds in LLF. In this study, one phenylethanoid together with seven PhGs were detected in the $80 \%$ methanol extract of processed-LLF. Hydroxytyrosol (2) was detected at $m / z$ 153.0556, with a fragment at $m / z 123$, which is due to the loss of the $\mathrm{CH}_{2} \mathrm{OH}$ group [11]. The presence of this phenylethanoid in LLF has been previously reported [35]. Hydroxytyrosol glucoside (3) was then eluted with a precursor $[\mathrm{M}-\mathrm{H}]^{-}$ion at $m / z 315.1089$ and fragmented in $\mathrm{MS}^{2}$ analysis to generate main ions at $m / z 123$ and 153 [11]. Compound 7 was easily identified as salidroside by comparing its retention time and $\mathrm{MS}^{2}$ spectrum with a commercial standard. Salidroside is a glucoside of tyrosol and it's responsible for the anti-osteoporosis and anti-oxidation actions of LLF $[35,36]$. According to our previous study, the content of salidroside increased a lot after the LLF was processed. Verbascoside (23), isoverbascoside (26), $\beta$-hydroxyverbascoside (13) and echinacoside (14) form a class of PhGs with caffeyl groups, thus the $\mathrm{MS}^{2}$ analyses of these four compounds showed the same fragments $(m / z 161,135$ and 179) characteristic of the caffeyl group [5,13]. Compounds $\mathbf{2 3}$ and $\mathbf{2 6}$ were only positional substitution isomers, and their fragmentation patterns were almost the same. Fortunately, compound 23 could be accurately identified as verbascoside by comparing its retention time with a standard. Therefore, compound $\mathbf{2 6}$ could be then proposed as isoverbascoside. Osmanthuside H (9) was detected at $m / z 431.1558$ with a major fragment at $m / z 299$, corresponding to the neutral loss of an apiofuranosyl group (132Da) from the precursor [M $-\mathrm{H}]^{-}$ ion. The ion at $m / z 299$ continued to produce a fragment at $m / z 119$ by the loss of the glucoside. Osmanthuside $\mathrm{H}$ had been previously reported in LLF [12] and the proposed fragmentation scheme is shown in Figure 3.

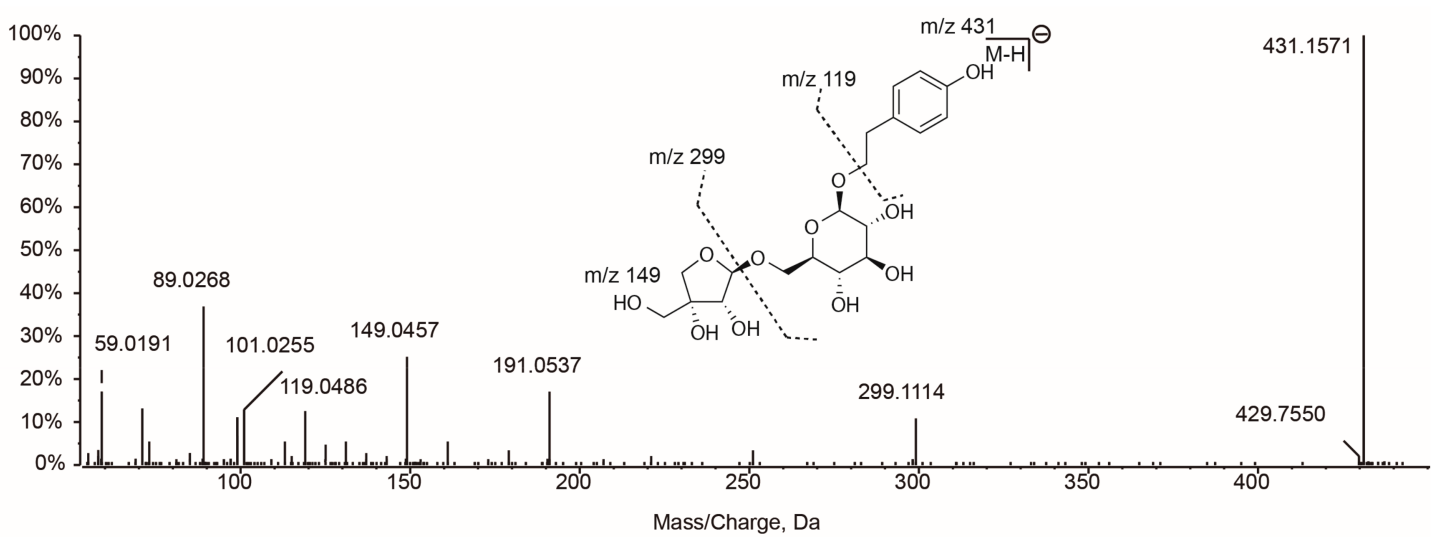

Figure 3. TOF-MS ${ }^{2}$ spectrum and fragmentation scheme proposed for osmanthuside $\mathrm{H}$.

\subsection{Identification of Flavonoids 16, 18, 20, 24, 28, 33, 39}

As many as eight flavonoids, including six flavones, one flavonol and one isoflavone, were identified in the $80 \%$ methanol extract of processed-LLF. Luteolin-7-O-glucoside (20) was detected at $m / z$ 447.0934, with a base peak at $m / z 285$ in its MS $^{2}$ spectrum, corresponding to free luteolin. In addition, free luteolin (33) was also extracted with main $\mathrm{MS}^{2}$ fragments at $m / z 133$ and 151, which were the result of a notable Ret-Diels-Alder (RDA) cleavage. The presence of this flavone and its glucoside was confirmed using commercial standards. Compound 39 was definitely identified as apigenin by comparison with the authentic substance. Compound 28 exhibited a precursor [M $-\mathrm{H}]^{-}$ ion at $m / z 431.0983$ with $\mathrm{MS}^{2}$ fragments at $m / z 268$ and 269, indicating the existence of apigenin aglycone [17]. This compound was thus tentatively identified as apigenin-7-O-glucoside (cosmosiin), which had been reported previously in Ligustrum lucidum Ait [37]. Compounds 16, 18 and 24 presented precursor ions at $m / z 609.1466,593.1511$ and 577.1557, respectively, and strong signals for the aglycones were observed in their $\mathrm{MS}^{2}$ spectra $(\mathbf{1 6}: \mathrm{m} / z$ 301; 18: $\mathrm{m} / \mathrm{z} 285 ; \mathbf{2 4 :} \mathrm{m} / \mathrm{z} 269)$ produced through the loss of a rutinosyl moiety (308 Da). By comparing their molecular formulas and fragmentation patterns 
with those reported in the literature [8,14,16], compounds $\mathbf{1 6}$ and $\mathbf{2 4}$ could be tentatively identified as quercetin-3-O-rutinoside (rutin) and apigenin-7-O-rutinoside, respectively, both of which had been isolated from Ligustrum lucidum Ait. [37,38]. As for compound 18, the ion at $m / z 285$ could be assigned as luteolin or kaempferol aglycone. According to reference [15], luteolin-7-O-rutinoside eluted before luteolin-7-O-glucoside, while kaempferol-3-O-rutinoside eluted after luteolin-7-O-glucoside under reversed phase liquid chromatography (RP-LC) conditions. Luteolin-7-O-glucoside had been accurately proposed for compound $20(\mathrm{RT}=15.28 \mathrm{~min})$. Considering the shorter retention time ( $\mathrm{RT}=14.78 \mathrm{~min}$ ), compound 18 could be thus tentatively identified as luteolin-7-O-rutinoside, which had been already reported in other plants belonging to the genus Ligustrum. whilst it is reported here for the first time in LLF [39].

\subsection{Identification of Iridoids $4-6,8,10-12,15,17,19,21,22,25,27,29-32,34-38,40,41$}

In most cases, the term iridoid is used to name a wide group of monoterpenes as well as glucoside derivatives, whose structure is in the general cyclopentanopyran form. Cleavage of a bond in the cyclopentane ring of iridoids gives rise to a subclass known as secoiridoids. LLF represents a rich source of iridoids, including cyclopentane iridoids and secoiridoids [3]. As many as 25 compounds of this type were present in processed-LLF, including 24 secoiridoids and one cyclopentane iridoid (loganic acid).

Loganic acid (5) was detected by a precursor $[\mathrm{M}-\mathrm{H}]^{-}$ion at $m / z 375.1300$, and its $\mathrm{MS}^{2}$ spectrum gave ions at $m / z 331,169$ and 151 . The fragment at $m / z 331$ could be justified by decarboxylation from the precursor ion, while the other two fragments represented the continued loss of glucose moieties and subsequent dehydration, respectively $[11,19]$. Compounds $4(\mathrm{RT}=5.50 \mathrm{~min})$ and $\mathbf{1 1}(\mathrm{RT}=8.57 \mathrm{~min})$ were both detected at $m / z 389.1093$ with almost the same fragmentation profile $(m / z 345,227,209$, 183,165 and 121). The fragment at $m / z 227$ was due to the loss of a hexose residue (162Da), which subsequently formed the fragment at $m / z 183$ by a neutral loss of $\mathrm{CO}_{2}$. The fragment at $m / z 183$ could gave ions at $m / z 165$ and 121 by successive loss of $\mathrm{H}_{2} \mathrm{O}$ and $\mathrm{CO}_{2}$. The above information indicated the presence of oleoside isomers [13,18], which had two carboxylic groups and a hexose in their structure. Oleoside and secologanoside had been reported in the genus Ligustrum [40]. Based on high mass accuracy $(<5 \mathrm{ppm})$ and the typical fragmentation profile, Compound 4 was tentatively identified as oleoside, while compound $\mathbf{1 1}$ was proposed as secologanoside, which was shown to elute after oleoside under RP-LC conditions [41]. Two isomers 10 and 12 exhibited a deprotonated ion at $m / z$ 403.1246, corresponding to $\mathrm{C}_{17} \mathrm{H}_{24} \mathrm{O}_{11}$. Identical fragmentation profile $(m / z 223,179,119$ and 89) were obtained by ESI-Q-TOF-MS ${ }^{2}$, indicating the presence of oleoside 11-methyl ester and its isomer [8,11,22], which had been previously reported in LLF [42]. Compound 21 was tentatively identified as elenolic acid, based on the intense ion at $m / z 241.0724$ and a strong signal at $m / z 139$ in its $\mathrm{MS}^{2}$ spectrum $[27,28]$.

Compound 30 exhibited a pseudomolecular ion at $m / z 539.1773$ with several fragments at $m / z 377$, $307,275,223,149$, and 139, which were characteristic for oleuropein $[8,11,29]$. As far as we knew, oleuropein had been isolated from LLF and showed strong antioxidant effects on free radical induced hemolysis of RBC [43]. Besides, oleuropein aglycone (41) was also observed by ESI-Q-TOF-MS with a precursor ion at $m / z 377.1243$ and products ions at $m / z 307,275,149$ and 139 [27,29,32]. Other oleuropein derivatives such as oleuropeinic acid (17) and hydroxyloleuropein (15) were also found in the $80 \%$ methanol extract of processed-LLF [8,23-25]. In their $\mathrm{MS}^{2}$ spectra, they both gave a base peak at $m / z 151$, which could be assigned to the phenolic moiety. Compound 34 showed a pseudomolecular ion at $m / z 523.1820$ with fragments at $m / z 361,291$ and 259. The fragment at $m / z 361$ was due to the loss of glucose, while the other two fragments were produced by the successive loss of $\mathrm{C}_{4} \mathrm{H}_{6} \mathrm{O}$ and $\mathrm{CH}_{3} \mathrm{OH}$. These fragments were all 16 mass units less than those of oleuropein, indicating the structure of compound $\mathbf{3 4}$ was short a hydroxyl group compared to oleuropein. Based on the fragments pattern and available references $[8,10,31]$, compound 34 was thus tentatively identified as ligustroside. 
Furthermore, a ligustroside derivative named ligustrosidic acid (27) was also detected in processed-LLF, and both of the two compounds had been already reported in LLF [3,42].

Specnuezhenide (syn. nuezhenide, 25) is known as one of the most abundant constituents in LLF, and typical fragments $(m / z 523,453,299$ and 223) was observed characteristic for this secoirdiod glucoside [22,44]. The fragment at $m / z 523$ was due to the neutral loss of glucoside, while the fragment at $m / z 299$ could be assigned as the tyrosol glucoside moiety in its structure (Figure 4 ). Furthermore, the identification of this compound was totally confirmed by an authentic standard. Two isomers of nuezhenide (compounds 22 and 29) were found in the $80 \%$ methanol extract of processed-LLF and they exhibited an identical fragmentation profile $(\mathrm{m} / \mathrm{z} 523,453,299$ and 223). Compound 29 was then tentatively identified as isonuezhenide based on the later retention time than nuezhenide [26], while it was difficult to ascertain the structure of compound 22 due to the absence of available references. Compound 19 showed a strong signal at $m / z 315$ in its MS $^{2}$ spectrum, indicating a hydroxytyrosol glucoside moiety in its structure [26]. Combined with other fragments $(m / z 539,469$, and 437), compound 19 can be tentatively identified as neonuezhenide which owns an extra hydroxyl group than nuezhenide. Compound 32 was tentatively identified as lucidumoside $C$ based on the high accuracy $m / z$ value at 583.2035 with a ppm as low as 0.5 , and the typical fragmentation pattern $(\mathrm{m} / \mathrm{z}$ 537, 403, 223 and 151) was in accordance with [8]. Neonuezhenide (19) and lucidumoside C (32) are two reported antioxidative glucosides from LLF, and they both showed strong antioxidant effects on free radical induced hemolysis of red blood cells [43].

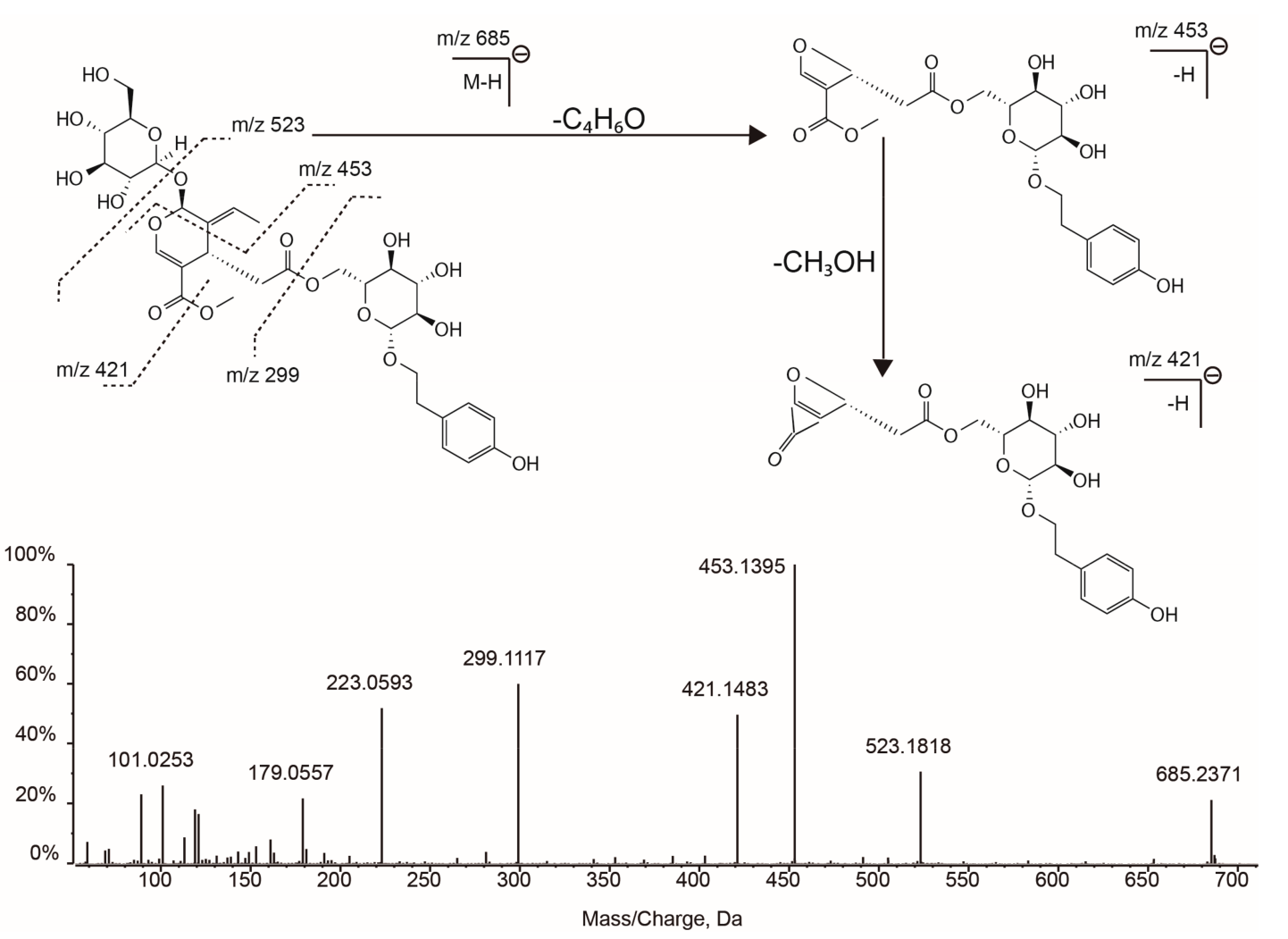

Figure 4. TOF-MS² spectrum and fragmentation scheme proposed for specnuezhenide.

The Extracted Ion Chromatogram (EIC) at $m / z 1071.3562$ displayed four peaks $(\mathbf{3 1}, \mathbf{3 5}, \mathbf{3 7}, \mathbf{3 8})$ with almost the same fragmentation profiles $(m / z 909,839,685,523,453,403,385)$ characteristic for G13 and its isomers $[13,30]$, which were possibly nuezhenide with an additional oleoside 11-methyl ester moiety. The fragments at $m / z 685$ and 403 could be assigned to the deprotonated ions of nuezhenide and oleoside 11-methyl ester, respectively. G13 was once isolated and identified from LLF, and it was reported to be responsible for the antiosteoporotic bioactivity of LLF [35]. Under RP-LC conditions, 
G13 was shown to be eluted after oleuropein but before ligstroside [45]. Based on above information, G13 could be proposed as compound 31. Three candidates, compounds 35, 37 and 38, were selected for these G13 isomers, i.e., oleonuezhenide, ligusides A and B, respectively [46]. However, it is impossible to distinguish between them without the use of other analysis methods (e.g., NMR).

In addition to the above mentioned secoiridiods, another four were also found in processed-LLF, i.e., nuezhenidic acid (6), nuezhenal A (8), 6'-O-trans-cinnamoyl-8-epikingisidic acid (36) and $6^{\prime}$-O-cis-cinnamoyl-8-epikingisidic acid (40). Nuezhenidic acid (6) is a reported secoiridiod glucoside in LLF [20], the content of which was demonstrated to have a significant increase after the LLF being processed with wine [47]. Nuezhenal A (8) and the 6'-O-cinnamoyl-8-epikingisidic acid isomers 36 and 40 are three recently discovered secoiridiod glucosides from LLF [21], and there are no references about them. These compounds were just tentatively identified base on the high mass accuracy $(\mathrm{ppm}<5)$.

\subsection{Identification of Triterpenoids $\mathbf{4 2}-\mathbf{5 0}$}

Besides the abovementioned compounds, a total of nine triterpenoids were also detected in the $80 \%$ methanol extract of processed-LLF. For most of these compounds, only intense precursor ions were obtained by ESI-Q-TOF-MS and it was difficult for the precursor ions to generate more MS $^{2}$ fragments under the conditions used. Thus, the identification of triterpenoids was mainly based on the high mass accuracy $(<5 \mathrm{ppm})$ and the knowledge that these compounds were previously reported to exist in LLF. Triterpenoids are an important type of compounds in LLF, including a pair of isomers named oleanolic acid and ursolic acid which have enjoyed good popularity for their hepatoprotective effects for both chronic liver fibrosis and acute liver injury induced by various chemical substances [48]. The EIC of the standard solution showed one peak for this two compounds with a retention time at $44.31 \mathrm{~min}$. Compound 49 was eluted at $44.35 \mathrm{~min}$ and exhibited the same spectrum as the authentic substances, which was thus proposed as oleanolic acid or ursolic acid. Similarly, compound 50 was identified as acetyloleanolic acid or acetylursolic acid due to the 42 unit heavier mass than compound 49. Compounds 43, 44 and 45 were tentatively identified as $19 \alpha$-hydroxyursolic acid (pomolic acid), $2 \alpha$-hydroxyoleanolic acid (maslinic acid) and $2 \alpha$-hydroxyursolic acid (colosic acid), respectively. These compounds are three isomers previously reported to exist in $\operatorname{LLF}[3,33]$ and their identification was based on their elution order under the RP-LC conditions [49]. Compound 42 was tentatively characterized as tormentic acid [33] also named $2 \alpha, 19 \alpha$-Dihydroxyursolic acid, which has an additional hydroxyl group than compounds 43 and 45 . Compound 48 gave a deprotonated ion at $m / z 513.3579$ and yielded major fragment ions at $m / z 495$ and 453 by elimination of a molecule of $\mathrm{H}_{2} \mathrm{O}$ and $\mathrm{CH}_{3} \mathrm{COOH}$, respectively. Therefore, compound 48 was tentatively proposed as $19 \alpha$-hydroxy-3-acetylursolic acid in LLF [34]. In addition, a pair of cis-trans-isomers named $3 \beta$-O-cis-p-coumaroyl-maslinic acid and $3 \beta-O$-trans- $p$-coumaroylmaslinic acid were also detected in processed-LLF, and they were tentatively proposed as compounds 46 and 47 , respectively. The intense signal at $m / z 145$ obtained by ESI-Q-TOF-MS ${ }^{2}$ indicated the presence of the coumaroyl moiety, and the two isomers were distinguished by the eluting orders reported in reference [50].

\subsection{Other Compounds}

Finally, a cyclohexanecarboxylic acid identified as quinic acid was also detected in the sample solution.

\section{Materials and Methods}

\subsection{Chemicals and Reagents}

HPLC-grade acetonitrile and formic acid used for LC-MS analysis were supplied by Merck KGaA (Darmstadt, Germany) and Anaqua Chemicals Supply Inc. (Houston, TX, USA), respectively. Deionized water was obtained from a Milli-Q water purification system (Millipore, Bedford, MA, USA). Methanol (HPLC grade) used for sample preparation was purchased from Hanbon Sci. \& Tech. (Huaian, China). Standards of oleanolic acid, ursolic acid, luteolin, apigenin, luteolin-7-O-glucoside 
and verbascoside, were purchased from National Institutes for Food and Drug Control (Beijing, China). Salidroside and specnuezhenide were obtained from Chengdu Push Bio-Technology Co., Ltd., (Chengdu, China).

As adjuvant material for crude drug processing, rice wine was purchased from Kuai Ji Shan Shaoxing Wine Co., Ltd. (Shaoxing, China). Rice wine is an alcoholic drink with an alcohol content of $15 \%(v / v)$, made from rice and traditionally consumed in East Asia, Southeast Asia, and South Asia. There are many types of rice wine, of which Shaoxing wine is probably the best known Chinese rice wine.

\subsection{Plant Materials and Processing}

LLF was purchased from Hetian Chinese Medicine Co., Ltd., (Tongling, China) and identified by Professor Qinan Wu (School of Pharmacy, Nanjing University of Chinese Medicine, Nanjing, China). The crude LLF was processed as follows to generate processed-LLF [51]: $100 \mathrm{~g}$ of LLF was put into a stainless steel box, mixed with $30 \mathrm{~g}$ of rice wine, steamed over boiling water for $8 \mathrm{~h}$, dried at $50{ }^{\circ} \mathrm{C}$ in an air blowing thermostatic oven and then stored in a cool and dry place.

\subsection{Sample and Standard Solution Preparation}

The processed-LLF was crushed into powder and $0.6 \mathrm{~g}$ accurately weighed into a $50 \mathrm{~mL}$ flask with $25 \mathrm{~mL}$ of $80 \%$ methanol-water $(v / v)$. It was extracted in an ultrasonic bath at room temperature for $30 \mathrm{~min}$. The extract was centrifuged at 15,000 rpm for $10 \mathrm{~min}$ and the supernatant was used for LC/MS analysis. All eight standards (oleanolic acid, ursolic acid, luteolin, apigenin, luteolin-7-O-glucoside, verbascoside, salidroside and specnuezhenide), were dissolved in $80 \%$ methanol-water $(v / v)$ at a concentration of $100 \mu \mathrm{g} / \mathrm{mL}$ to make a standard solution.

\subsection{UHPLC-ESI-QTOF-MS System and Conditions}

Chromatographic analysis was performed on a Shimadzu UHPLC system (Kyoto, Japan) equipped with LC-20AD XR pumps, CTO-20AC column oven and SIL-20A XR auto injector. Components were separated on a Poroshell 120 EC-C18 $(2.1 \mathrm{~mm} \times 100 \mathrm{~mm}, 2.7 \mu \mathrm{m})$ at $30{ }^{\circ} \mathrm{C}$ with a flow rate of $0.3 \mathrm{~mL} / \mathrm{min}$. The mobile phase was composed of $0.1 \%(v / v)$ formic acid in water (solvent A) and acetonitrile (solvent B). The linear gradient elution program was as follows: $0-10 \mathrm{~min}, 2-15 \% \mathrm{~B}$; 10-30 min, 15-25\% B; 30-35 min, 25-35\% B; 35-40 min, 35-75\% B; 40-46 min, 75-95\% B; 46-48 min, $95-2 \% \mathrm{~B}$. The injection volume was set at $4 \mu \mathrm{L}$.

MS analysis was performed on a triple TOF 5600 System equipped with an electrospray ionization (ESI) interface (AB SCIEX, Concord, ON, Canada). The MS acquisition was operated in both negative and positive ion mode, and mass rang was set at $m / z$ 50-1500. The parameters were set as follows in negative ion mode: ion spray voltage: $-4500 \mathrm{eV}$; ion source temperature (TEM): $550{ }^{\circ} \mathrm{C}$; declustering potential (DP): $-100 \mathrm{eV}$; curtain gas (N2): 40 psi; atomizing gas (N2): 55 psi; auxiliary gas (N2): 55 psi. Information-dependent acquisition (IDA) was used to trigger acquisition of MS/MS spectra with collision energy (CE) and collision energy spread (CES) fixed at $-40 \mathrm{eV}$ and $20 \mathrm{eV}$ respectively. When it comes to the positive ion mode, the conditions were the same but some voltages can change their polarity to positive. Meanwhile, an automated calibration delivery system (CDS) could regulate the MS and MS/MS data automatically.

\subsection{Data Processing}

Data acquisition and processing was carried out using software Analyst, Peak View 1.2 with the application of XIC manager and Formula Finder (AB SCIEX). The SciFinder database (https://scifinder.cas.org) was employed to get the information of previously reported compounds in LLF. 


\section{Conclusions}

In this study, a powerful analytical method has been used to characterize 51 compounds (one phenylethanoid, seven phenylethanoid glycosides, eight flavonoids, 25 iridoids, nine triterpenoids and one cyclohexanecarboxylic acid) in the $80 \%$ methanol extract of processed-LLF. Four compounds-quercetin-3,4'-diglucoside, luteolin-7-O-rutinoside, oleoside and secologanoside-were identified for the first time in processed-LFF. In addition, the MS fragmentation of the compounds were interpreted and summarized in details. Hence, this study offered a reliable reference for the quality assessment and further research of processed-LLF and crude LLF. The established UHPLC-ESI-Q-TOF-MS method can be applied as a template for the systematic analysis of other complicated herbal medicines. Our next work is to search for differences between the compounds identified in crude and processed materials using a chemometric method.

Acknowledgments: This work was financially supported by National Natural Science Foundation of China (Grant No. 81373972 and 81573554), A Project Funded by the Priority Academic Program Development of Jiangsu Higher Education Institutions (PAPD), and Qing Lan Project of Jiangsu Province.

Author Contributions: H.L., Q.N.L and B.H.B performed the experiments; W.F.Y., J.X. and H.L. analyzed the data; M.Q.S., Y.D.C and F.F.C. contributed reagents/materials/analysis tools; and W.F.Y., L.Z and A.W.D. wrote the paper.

Conflicts of Interest: The authors declare no conflict of interest.

\section{References}

1. Gu, G. Shen Nong's Herbal Classic; Huaxia Publishing House: Beijing, China, 1999; p. 43.

2. Chinese Pharmacopoeia Commission. Pharmacopoeia of the People's Republic of China; China Medical Science Press: Beijing, China, 2015; Volume I, p. 45.

3. Gao, L.; Li, C.; Wang, Z.; Liu, X.; You, Y.; Wei, H.; Guo, T. Ligustri lucidi fructus as a traditional Chinese medicine: A review of its phytochemistry and pharmacology. Nat. Prod. Res. 2015, 29, 493-510. [CrossRef] [PubMed]

4. Zhan, F.; Zhang, X.; Jiang, H.; Zhao, Z. Assay of Ligustrum lucidum Ait. with and without steaming with wine by HPLC-ESI/MS. Chin. Tradit. Pat. Med. 2013, 35, 2707-2710.

5. Han, L.; Boakye-Yiadom, M.; Liu, E.; Zhang, Y.; Li, W.; Song, X.; Fu, F.; Gao, X. Structural characterisation and identification of phenylethanoid glycosides from Cistanches deserticola YC Ma by UHPLC/ESI-QTOF-MS/MS. Phytochem. Anal. 2012, 23, 668-676. [CrossRef] [PubMed]

6. Amessis-Ouchemoukh, N.; Abu-Reidah, I.M.; Quirantes-Piné, R.; Rodríguez-Pérez, C.; Madani, K.; Fernández-Gutiérrez, A.; Segura-Carretero, A. Tentative characterisation of iridoids, phenylethanoid glycosides and flavonoid derivatives from Globularia alypum L. (Globulariaceae) leaves by LC-ESI-QTOF-MS. Phytochem. Anal. 2014, 25, 389-398. [CrossRef] [PubMed]

7. Liu, M.H.; Tong, X.; Wang, J.X.; Zou, W.; Cao, H.; Su, W.W. Rapid separation and identification of multiple constituents in traditional Chinese medicine formula Shenqi Fuzheng Injection by ultra-fast liquid chromatography combined with quadrupole-time-of-flight mass spectrometry. J. Pharm. Biomed. Anal. 2013, 74, 141-155. [CrossRef] [PubMed]

8. Quirantes-Piné, R.; Lozano-Sánchez, J.; Herrero, M.; Ibáñez, E.; Segura-Carretero, A.; Fernández-Gutiérrez, A. HPLC-ESI-QTOF-MS as a powerful analytical tool for characterizing phenolic compounds in olive leaf extracts. Phytochem. Anal. 2013, 24, 213-223. [CrossRef] [PubMed]

9. Yang, S.T.; Wu, X.; Rui, W.; Guo, J.; Feng, Y.F. UPLC/Q-TOF-MS analysis for identification of hydrophilic phenolics and lipophilic diterpenoids from Radix Salviae Miltiorrhizae. Acta Chromatogr. 2015, 27, 711-728. [CrossRef]

10. Song, J.; Zhao, L.; Rui, W.; Guo, J.; Feng, Y. Identification and Fragmentation Pattern Analysis of Iridoid Glycosides Fromfructus Ligustri Lucidiby UPLC/ESI-QTOF-MS. J. Liq. Chromatogr. Relat. Technol. 2014, 37, 1763-1770. [CrossRef] 
11. Peralbo-Molina, A.; Priego-Capote, F.; Luque de Castro, M.D. Tentative identification of phenolic compounds in olive pomace extracts using liquid chromatography-tandem mass spectrometry with a quadrupole-quadrupole-time-of-flight mass detector. J. Agric. Food. Chem. 2012, 60, 11542-11550. [CrossRef] [PubMed]

12. Huang, X.; Yin, Z.; Ye, W.; Shen, W. Chemical constituents from fruits of Ligustrum lucidum. China J. Chin. Mater. Med. 2010, 35, 861-864.

13. Klen, T.J.; Wondra, A.G.; Vrhovsek, U.; Vodopivec, B.M. Phenolic profiling of olives and olive oil process-derived matrices using UPLC-DAD-ESI-QTOF-HRMS analysis. J. Agric. Food. Chem. 2015, 63, 3859-3872. [CrossRef] [PubMed]

14. Taamalli, A.; Arraez-Roman, D.; Ibanez, E.; Zarrouk, M.; Segura-Carretero, A.; Fernandez-Gutierrez, A. Optimization of microwave-assisted extraction for the characterization of olive leaf phenolic compounds by using HPLC-ESI-TOF-MS/IT-MS(2). J. Agric. Food. Chem. 2012, 60, 791-798. [CrossRef] [PubMed]

15. Ola, S.S.; Catia, G.; Marzia, I.; Vincieri, F.F.; Akindahunsi, A.A.; Mulinacci, N. HPLC/DAD/MS characterisation and analysis of flavonoids and cynnamoil derivatives in four Nigerian green-leafy vegetables. Food Chem. 2009, 115, 1568-1574. [CrossRef]

16. Cuyckens, F.; Rozenberg, R.; de Hoffmann, E.; Claeys, M. Structure characterization of flavonoid O-diglycosides by positive and negative nano-electrospray ionization ion trap mass spectrometry. J. Mass Spectrom. 2001, 36, 1203-1210. [CrossRef] [PubMed]

17. Hvattum, E.; Ekeberg, D. Study of the collision-induced radical cleavage of flavonoid glycosides using negative electrospray ionization tandem quadrupole mass spectrometry. J. Mass Spectrom. 2003, 38, 43-49. [CrossRef] [PubMed]

18. Fu, S.; Arraez-Roman, D.; Segura-Carretero, A.; Menendez, J.A.; Menendez-Gutierrez, M.P.; Micol, V.; Fernandez-Gutierrez, A. Qualitative screening of phenolic compounds in olive leaf extracts by hyphenated liquid chromatography and preliminary evaluation of cytotoxic activity against human breast cancer cells. Anal. Bioanal. Chem. 2010, 397, 643-654. [CrossRef] [PubMed]

19. Sun, H.; Liu, M.; Lin, Z.; Jiang, H.; Niu, Y.; Wang, H.; Chen, S. Comprehensive identification of 125 multifarious constituents in Shuang-huang-lian powder injection by HPLC-DAD-ESI-IT-TOF-MS. J. Pharm. Biomed. Anal. 2015, 115, 86-106. [CrossRef] [PubMed]

20. Wu, L.; Xiang, T.; Hou, B.; Liang, W.; Yin, S.; Zhou, X. Chemical constituents from fruits of Ligustrum lucidum. Acta Bot. Sin. 1997, 40, 83-87.

21. Aoki, S.; Honda, Y.; Kikuchi, T.; Miura, T.; Sugawara, R.; Yaoita, Y.; Kikuchi, M.; Machida, K. Six New Secoiridoids from the Dried Fruits of Ligustrum lucidum. Chem. Pharm. Bull. 2012, 60, 251-256. [CrossRef] [PubMed]

22. Bianco, A.; Buiarelli, F.; Cartoni, G.; Coccioli, F.; Jasionowska, R.; Margherita, P. Analysis by liquid chromatography-tandem mass spectrometry of biophenolic compounds in olives and vegetation waters, Part I. J. Sep. Sci. 2003, 26, 409-416. [CrossRef]

23. Tóth, G.; Alberti, Á.; Sólyomváry, A.; Barabás, C.; Boldizsár, I.; Noszál, B. Phenolic profiling of various olive bark-types and leaves: HPLC-ESI/MS study. Ind. Crops Prod. 2015, 67, 432-438. [CrossRef]

24. Rubio-Senent, F.; Lama-Munoz, A.; Rodriguez-Gutierrez, G.; Fernandez-Bolanos, J. Isolation and identification of phenolic glucosides from thermally treated olive oil byproducts. J. Agric. Food. Chem. 2013, 61, 1235-1248. [CrossRef] [PubMed]

25. Guo, N.; Yu, Y.; Ablajan, K.; Li, L.; Fan, B.; Peng, J.; Yan, H.; Ma, F.; Nie, Y. Seasonal variations in metabolite profiling of the fruits of Ligustrum lucidum Ait. Rapid Commun. Mass Spectrom. 2011, 25, 1701-1714. [CrossRef] [PubMed]

26. Tóth, G.; Barabás, C.; Tóth, A.; Kéry, Á.; Béni, S.; Boldizsár, I.; Varga, E.; Noszál, B. Characterization of antioxidant phenolics in Syringa vulgaris L. flowers and fruits by HPLC-DAD-ESI-MS. Biomed. Chromatogr. 2016, 30, 923-932. [CrossRef] [PubMed]

27. Garcia-Villalba, R.; Carrasco-Pancorbo, A.; Oliveras-Ferraros, C.; Vazquez-Martin, A.; Menendez, J.A.; Segura-Carretero, A.; Fernandez-Gutierrez, A. Characterization and quantification of phenolic compounds of extra-virgin olive oils with anticancer properties by a rapid and resolutive LC-ESI-TOF MS method. J. Pharm. Biomed. Anal. 2010, 51, 416-429. [CrossRef] [PubMed] 
28. Kanakis, P.; Termentzi, A.; Michel, T.; Gikas, E.; Halabalaki, M.; Skaltsounis, A.L. From olive drupes to olive oil. An HPLC-orbitrap-based qualitative and quantitative exploration of olive key metabolites. Planta Med. 2013, 79, 1576-1587. [CrossRef] [PubMed]

29. Savarese, M.; Demarco, E.; Sacchi, R. Characterization of phenolic extracts from olives (Olea europaea cv. Pisciottana) by electrospray ionization mass spectrometry. Food Chem. 2007, 105, 761-770. [CrossRef]

30. Garcia-Villalba, R.; Tomas-Barberan, F.A.; Fanca-Berthon, P.; Roller, M.; Zafrilla, P.; Issaly, N.; Garcia-Conesa, M.T. Targeted and untargeted metabolomics to explore the bioavailability of the secoiridoids from a seed/fruit extract (Fraxinus angustifolia Vahl) in human healthy volunteers: A preliminary study. Molecules 2015, 20, 22202-22219. [CrossRef] [PubMed]

31. Michel, T.; Khlif, I.; Kanakis, P.; Termentzi, A.; Allouche, N.; Halabalaki, M.; Skaltsounis, A.-L. UHPLC-DAD-FLD and UHPLC-HRMS/MS based metabolic profiling and characterization of different Olea europaea organs of Koroneiki and Chetoui varieties. Phytochem. Lett. 2015, 11, 424-439. [CrossRef]

32. Fu, S.; Arráez-Román, D.; Menendez, J.A.; Segura-Carretero, A.; Fernández-Gutiérrez, A. Characterization of isomers of oleuropein aglycon in olive oils by rapid-resolution liquid chromatography coupled to electrospray time-of-flight and ion trap tandem mass spectrometry. Rapid Commun. Mass Spectrom. 2009, 23, 51-59. [CrossRef] [PubMed]

33. Zhang, T.; Tu, F.; Dai, Y.; Yao, X. Chemical Constituents of Ligustrum lucidum. China Pharm. 2011, 22, 2931-2933.

34. Feng, J.; Feng, Z.; Wang, J.; Cui, Y. Study on the Triterpenoids from the Fruits of Ligustrum lucidum. J. Chin. Med. Mater. 2011, 34, 1540-1544.

35. Chen, Q.; Yang, L.; Zhang, G.; Wang, F. Bioactivity-guided isolation of antiosteoporotic compounds from Ligustrum lucidum. Phytother. Res. 2013, 27, 973-979. [CrossRef] [PubMed]

36. Ju, H.Y.; Chen, S.C.; Wu, K.J.; Kuo, H.C.; Hseu, Y.C.; Ching, H.; Wu, C.R. Antioxidant phenolic profile from ethyl acetate fraction of Fructus Ligustri Lucidi with protection against hydrogen peroxide-induced oxidative damage in SH-SY5Y cells. Food Chem. Toxicol. 2012, 50, 492-502. [CrossRef] [PubMed]

37. Xu, X.; Yang, N.; Qian, S.; Xie, N.; Yu, M.; Duan, J. Stduy on flavonoids in Ligustrum lucidum. China J. Chin. Mater. Med. 2007, 30, 538-540.

38. Long, F.; Deng, L.; Chen, Y. Study on the chemical constituents in the flowers of Ligustrum lucium. West China J. Pharm. Sci. 2011, 26, 97-100.

39. Nagy, M.; Krizkova, L.; Mucaji, P.; Kontsekova, Z.; Sersen, F.; Krajcovic, J. Antimutagenic activity and radical scavenging activity of water infusions and phenolics from ligustrum plants leaves. Molecules 2009, 14, 509-518. [CrossRef] [PubMed]

40. Jensen, S.R.; Franzyk, H.; Wallander, E. Chemotaxonomy of the Oleaceae: iridoids as taxonomic markers. Phytochemistry 2002, 60, 213-231. [CrossRef]

41. Obied, H.K.; Bedgood, D.R., Jr.; Prenzler, P.D.; Robards, K. Chemical screening of olive biophenol extracts by hyphenated liquid chromatography. Anal. Chim. Acta 2007, 603, 176-189. [CrossRef] [PubMed]

42. He, Z.; Dong, H.; Xu, H.; Ye, W.; Sun, H.; But, P.P.-H. Secoiridoid constituents from the fruits of Ligustrum lucidum. Phytochemistry 2001, 56, 327-330. [CrossRef]

43. He, Z.; But, P.P.-H.; Chan, T.-W.D.; Dong, H.; Xu, H.; Lau, C.; Sun, H. Antioxidative Glucosides from the Fruits of Ligustrum lucidum. Chem. Pharm. Bull. 2001, 49, 780-784. [CrossRef] [PubMed]

44. Silva, S.; Gomes, L.; Leitão, F.; Bronze, M.; Coelho, A.V.; Boas, L.V. Secoiridoids in olive seed: characterization of nüzhenide and 11-methyl oleosides by liquid chromatography with diode array and mass spectrometry. Grasas Aceites 2010, 61, 157-164. [CrossRef]

45. Jiang, Q.; Jiang, H.; Li, H.; Zhang, X. Contents dynamic changes of four secoiridoid glycosides under steaming time spans with wine in Ligubtrum lucidum Ait. Chin. Tradit. Pat. Med. 2014, 36, 2561-2564.

46. Huang, X.J.; Wang, Y.; Yin, Z.Q.; Ye, W.C. Two new dimeric secoiridoid glycosides from the fruits of Ligustrum lucidum. J. Asian Nat. Prod. Res. 2010, 12, 685-690. [CrossRef] [PubMed]

47. Li, Z.; Zhang, X.; Li, H.; Cui, W. Dynamic changes of neonuezhenide, oleoside-11-methyl ester and nuezhenidic acid in wine steaming process of Ligustri Lucidi Fructus. Chin. J. Exp. Tradit. Med. Formulae 2014, 20, 14-17.

48. Yim, T.K.; Wu, W.K.; Pak, W.F.; Ko, K.M. Hepatoprotective action of an oleanolic acid-enriched extract of Ligustrum lucidum fruits is mediated through an enhancement on hepatic glutathione regeneration capacity in mice. Phytother. Res. 2001, 15, 589-592. [CrossRef] [PubMed] 
49. Zhang, Y.; Zhang, C.; Ren, R.; Liu, R. Simultaneous determination of seven major triterpenoids in Pyrola decorata H. Andres by LC-MS method. Pharmazie 2012, 67, 822-826.

50. Masullo, M.; Montoro, P.; Autore, G.; Marzocco, S.; Pizza, C.; Piacente, S. Quali-quantitative determination of triterpenic acids of Ziziphus jujuba fruits and evaluation of their capability to interfere in macrophages activation inhibiting NO release and iNOS expression. Food Res. Int. 2015, 77, 109-117. [CrossRef]

51. Li, H.; Liu, Q.; Zhang, L.; Yao, W.; Ding, A. Optimization of processing technology of Ligustri Lucidi Fructus stewed with wine based on analytic hierarchy process and multi-index orthogonal test. Chin. Tradit. Herb. Drugs 2016, 47, 2832-2837.

Sample Availability: Samples of all the compounds are not available from the authors.

(C) 2017 by the authors. Licensee MDPI, Basel, Switzerland. This article is an open access article distributed under the terms and conditions of the Creative Commons Attribution (CC BY) license (http:/ / creativecommons.org/licenses/by/4.0/). 